\section{$\underset{\substack{\text { hommes } \\ \text { \& migrations }}}{ }$}

\section{Hommes \& migrations}

Revue française de référence sur les dynamiques

migratoires

1316 | 2017

L'islam en Europe

\title{
Koln Concert
}

Retour sur un « cohérent délire » postcolonial

\section{Andrea Brazzoduro}

\section{Q OpenEdition \\ 1 Journals}

\section{Édition électronique}

URL : http://journals.openedition.org/hommesmigrations/3795

DOI : 10.4000/hommesmigrations.3795

ISSN : 2262-3353

Éditeur

Musée national de l'histoire de l'immigration

\section{Édition imprimée}

Date de publication : 1 mars 2017

Pagination : 59-67

ISBN : 978-2-919040-37-7

ISSN : $1142-852 X$

Référence électronique

Andrea Brazzoduro, « Koln Concert », Hommes \& migrations [En ligne], 1316 | 2017, mis en ligne le 01 mars 2020, consulté le 18 septembre 2020. URL : http://journals.openedition.org/hommesmigrations/ 3795 ; DOI : https://doi.org/10.4000/hommesmigrations.3795 


\title{
KÖLN CONCERT \\ RETOUR SUR UN « COHÉRENT DÉLIRE » POSTCOLONIAL
}

Par ANDREA BRAZZODURO, Marie Curie Fellow en histoire à Trinity College, Oxford.

\author{
La vague d'agressions à caractère sexuel qui ont eu lieu en \\ Allemagne la nuit du 31 décembre 2015 a suscité une réception \\ complexe et clivante dans les médias d'Europe et du Maghreb. \\ Dans les jours qui suivent, un article de l'écrivain Kamel Daoud \\ déclenche une vive polémique portant sur l'analyse des causes \\ de cet événement attribué à des migrants extra-européens. \\ En retour, dix-neuf universitaires internationaux lui répondent \\ dans une tribune dénonçant sa vision des supposés agresseurs. \\ Au cœur de ces débats, se trouvent les assignations culturelles \\ et identitaires qui contribuent, en Europe, à pérenniser un \\ imaginaire postcolonial.
}

Un an après les violences de la Saint-Sylvestre en Allemagne (dans la ville de Cologne mais dénoncées également à Hambourg, Bielefeld, Dortmund, Düsseldorf, Francfort et Stuttgart), on ne sait pas encore ce qui s'est exactement passé cette nuit du 31 décembre 2015. Le procès concernant les incidents dénoncés à Hambourg s'est soldé, le $1^{\mathrm{er}}$ novembre 2016, par l'acquittement des présumés coupables, détenus pendant six mois : le tribunal a reconnu que les accusations et les preuves avaient été manipulées par la police $^{1}$. Fait grave, et très peu relaté par la presse, mais qui surtout accentue encore les ombres sur un épisode déjà assez sombre. Pour le verdict concernant la ville de Cologne, où le nombre d'agressions à caractère sexuel dénoncées a été le plus haut (autour de 600), il faudra attendre avril 2017.
La vérité judiciaire repose bien évidemment sur des procédures d'administration de la preuve qui diffèrent de celles propres à la recherche de la vérité historique. Ce jugement du tribunal de Hambourg affaibli toutefois l'hypothèse du «terrorisme sexuel » avancée par Éric Fassin, pourtant une des meilleures analyses disponibles sur le sujet, à savoir que les violences n'étaient pas du tout spontanées, expression d'une «culture » sous-jacente, mais bien au contraire organisées dans les différentes villes et coordonnées par une organisation bien précise : "Daesh (ou quelque autre organisation), en s'appuyant sur des bandes criminelles [...] a semé la terreur en vue d’infléchir le cours politique de l'Europe ${ }^{2}$ ».

Quels que soient les faits - rarement on a tant regretté l'adage positiviste sur « ce qui s'est réelle- 
ment passé »-, il est certain qu'il y aura un avant et un après Cologne dans les imaginaires qui informent notre présent. Après Cologne - a ainsi pu écrire Nadia Tazi -, " les fantasmes racistes ou islamophobes les plus insistants peuvent être vérifiés. Les pires abjections et les pathologies reprochées depuis toujours aux musulmans ont bien eu lieu un soir de fête : en l'obscur, les mains avides, la bestialité et l'injure sur les corps des jeunes Blanches ${ }^{3}$ ». D'ailleurs, après l'attentat du 19 décembre au marché de Berlin, Le Monde n'a pas hésité à mettre les deux événements sur le même plan : "Cologne l'avait annoncé, Berlin l'a confirmé : 2016, en Allemagne, marquera la fin d'une certaine insouciance ${ }^{4}$. »

Ces mots, ces discours ont déjà des effets qui sont tout sauf imaginaires, comme l'a par exemple démontré le tweet de la police de Cologne, publié le samedi 31 décembre 2016, à 23 h 08: «De nombreux "Nafris" [nordafrikanische Intensivtäter, nord-africains délinquants] ont été contrôlés à la gare centrale. » Au cours de la nuit, un dispositif de sécurité imposant - 1500 policiers mobilisés - a contrôlé l'identité de 674 personnes,

Quels que soient les faits

- rarement on a tant regretté

l'adage positiviste sur

«ce qui s'est réellement

passé »-, il est certain qu’il

y aura un avant et un après

Cologne dans les imaginaires qui informent notre présent. interdit à 190 d'accéder à la place du Dom, tandis que 92 ont été placées en garde à vue ${ }^{5}$. Toutes ces personnes n'ont pas été inquiétées par la police en raison d'un comportement criminel, voire répréhensible, mais par profilage racial.

C'est dans cette perspective, quand l'écart entre faits et discours a atteint son paroxysme, et que les mots sont en train de fabriquer des faits bien réels, qu'il semble utile de revenir, exactement une année après, sur la controverse déclenchée par les événements que l'on désigne couramment comme
« les violences de Cologne ». Il s'agit de revenir au début du débat qui les a accompagnées, provoqué par certaines interventions de Kamel Daoud. L'objectif, cependant, n'est pas de prendre parti pour ou contre Daoud, mais plutôt d'analyser la configuration discursive cristallisée dans un véritable phénomène d'hystérie collective qui, bien au-delà du cas de l'écrivain algérien, est symptomatique des stéréotypes et des préjugés qui informent l'ordre du discours occidental sur les musulmans ou, selon une désignation désormais commune, sur l'« islam ${ }^{6} »$.

\section{Quand un papier jette de I'huile sur le feu}

Kamel Daoud est désormais un nom familier aux lecteurs bien informés, largement au-delà du seul espace francophone. Journaliste né et résidant en Algérie, il s'est fait connaître au tournant des années 2000 grâce à une rubrique dans Le Quotidien d'Oran, journal dont il a été également le directeur. C'est dans les dernières années terribles du Front islamique du salut que Daoud détonne par son regard libre et son style caustique, en s'attaquant également au nouveau président «pacificateur », Abdelaziz Bouteflika, élu en 1999 sur un programme de «concorde civile ${ }^{7}$ ».

La même irrévérence anime deux interventions plus récentes qui ont eu une large diffusion : "Ce pourquoi je ne suis pas "solidaire" de Gaza ", publié à l'été 2014 dans Le Quotidien d'Oran et "Un Daesh qui a réussi », analyse impitoyable de l'Arabie saoudite et de ses alliés " historiques" occidentaux, publié dans The New York Times après les attentats du 13 novembre 2015 à Paris ${ }^{8}$. Ce qui a propulsé Daoud du Quotidien d'Oran au

3. Nadia Tazi, "Après Cologne ", in Esprit, n 5, mai 2016, p. 14. 4. Thomas Wieder, " 2016, la fin de l'insouciance ", in Le Monde, 31 décembre 2016. 5. Voir Thomas Wieder, «Allemagne : à Cologne, un réveillon sous contrôle pour ne pas revivre le cauchemar de 2015 ", in Le Monde, 2 janvier 2017. 6. Distinction reprise récemment aussi par Olivier Roy, "La mort fait partie du projet djihadiste ", entretien avec Nicolas Truong, in Le Monde, 13 octobre 2016 ; de manière plus générale, voir Olivier Roy, Le djihad et la mort, Paris, Seuil, 2016. 7. Sur le parcours de Daoud, voir Kaoutar Harchi, Je n'ai qu'une langue, ce n'est pas la mienne. Des écrivains à l'épreuve, Paris, Pauvert, 2016, en particulier le chapitre "Kamel Daoud: de la tentation islamiste au salut littéraire. La reconnaissance au prix du malentendu ". 8. "Ce pourquoi je ne suis pas "solidaire" de Gaza ", in Le Quotidien d'Oran, 13 juin 2014 ; "Saudi Arabia, an ISIS that has made it ", in The New York Times, 20 novembre 2015. 
New York Times a été la consécration mondiale arrivée avec son roman Meursault, contre-enquête (Barzakh, 2013 ; Actes Sud ; 2014 ; puis rapidement traduit en 29 langues), réécriture en contrepoint de L'Étranger de Camus avec les yeux d'Haroun, le frère de l'« Arabe », l'Algérien sans nom tué par Meursault sur une plage ensoleillée. Finaliste au Goncourt, le livre a enchaîné les succès (notamment en France: Prix François Mauriac, Prix des cinq continents de la francophonie, Prix Goncourt du premier roman...), et son auteur, invité par les universités les plus prestigieuses du monde (Oxford, Yale...), a reçu une pluie de récompenses, jusquau prix Jean-Luc Lagardère en qualité de meilleur journaliste de l'année pour sa rubrique dans Le Point, que le Premier ministre a tenu à lui remettre personnellement. C'est que Daoud n'est pas seulement un journaliste et écrivain à succès. En décembre 2014, un obscur imam d'Alger, Zeraoui Hamadache, a lancé sur Facebook une fatwa contre lui, l'accusant d' " apostasie » et de "sionisme ». Bien que Zeraoui soit une figure marginale, c'est sans aucun doute un incident grave et, en mars dernier, l'« imam » a été condamné par le tribunal d'Oran à six mois de prison. C'est ainsi, toutefois, que Daoud - qui n'a même pas voulu participer au procès - s'est retrouvé malgré lui embrigadé dans des batailles qui ont peu à voir avec l'Algérie.

Le 10 janvier, son article sur les violences de la nuit de la Saint-Sylvestre (" Le corps des femmes et le désir de liberté de ces hommes arrachés à leur terre ») ne sort pas dans le Quotidien d'Oran, mais dans le journal italien La Repubblica, puis le 31 janvier dans Le Monde9. L'emplacement de l'article n'est pas un aspect secondaire : il nous indique d'où on choisit de parler et donc à qui on s'adresse. Quand il parle des violences de Cologne, l'auteur du Meursault ne parle pas depuis l'Algérie aux Algériens, mais depuis l'Europe aux Européens ${ }^{10}$.

Daoud décide d'écrire alors que l'on a encore que très peu d'éléments sur ce qui s'est réellement passé, et que circulent déjà d' "incontestables " photographies et vidéos - s'étant avérées plus tard des faux - qui donnent libre cours au racisme ambiant, Decertain, il y a les plus ou moins explicite- 1170 plaintes pour ment, sur le vieux continent ${ }^{11}$. Pour évoquer l'atmosphère de ces jours, il ne faut pas penser aux néonazis de Pegida qui le 9 janvier, la veille de la publicaagressions, dont autour de 600 pour agression sexuelle et 2 pour viol : il s'agit sans doute d'un épisode de violence à connotation sexiste gravissime. tion de l'article de Daoud, ont participé à une manifestation avec le parti "Pro Koehln » s'étant terminée par des raids racistes (12 blessés). Il suffit de rappeler quelques titres de journaux classés au centre-gauche, tel le quotidien italien fondé par Eugenio Scalfari, La Repubblica, qui a publié le premier l'article de Daoud sur les violences de Cologne et titre pleine page : "Cologne, attaque planifiée : "Harcelez la femme blanche"12 ».

Compte tenu du contexte explosif, l'article de Daoud sur la nuit de la Saint-Sylvestre commence par une question particulièrement opportune : «Que s'est-il passé à Cologne? » Encore aujourd'hui, presque un an après, nous ne le savons pas exactement. De certain, il y a les 1170 plaintes pour agressions, dont autour de 600 pour agression sexuelle et 2 pour viol : il s'agit sans doute d'un épisode de violence à connotation sexiste gravissime. La majorité des arrestations n'a toutefois pas

9. "Colonia. Il corpo delle donne e il desiderio di libertà di quegli uomini sradicati dalla loro terra ", in la Repubblica, 10 janvier 2016 ; "Cologne, lieu de fantasmes ", in Le Monde, 31 janvier 2016. Toutes les traductions sont les miennes. 10. Une semaine après, Kamel Daoud publie en Algérie un article sur le même thème mais sensiblement différent : "La "colognisation" du monde ", in Le Quotidien d'Oran, 18 janvier 2016. II a été aussi remarqué que l'édition française du roman Meursault, contre-enquête diffère de l'édition algérienne : voir Alice Kaplan, "Making the stranger contemporary : Kamel Daoud's Meursault, contre-enquête ", in Lia Brozgal, Sara Kippur (dir.), Being Contemporary: French Literature, Culture, and Politics Today, Liverpool, Liverpool University Press, 2016, pp. 334-336. 11. Pour une excellente reconstruction des faits, en italien, voir Angelo Romano, Andrea Zitelli, « Colonia : i fatti, le indagini, le reazioni, il dibattito ", in www.valigiablu.it., 18 janvier 2016, http://www.valigiablu.it/colonia-i-fatti-le-indagini-lereazioni-il-dibattito/ (tous les sites Internet ont été consultés une dernière fois le 15 janvier 2017). 12. "Colonia, attacco pianificato "Molestate la donna bianca" ", in La Repubblica, 11 janvier 2016. 
entraîné de suites parce que les victimes n'ont pas reconnu leurs agresseurs. Le premier procès pour agression sexuelle a vu, faute de preuves, l'acquittement de l'accusé, un Algérien de 26 ans. Il y a eu, par contre, 9 condamnations pour vol. Les deux autres procès pour violence sexuelle qui se sont déroulés en juillet ont vu les suspects, un Irakien et un Algérien, condamnés à une mise à l'épreuve : les victimes ne les ont pas reconnus ${ }^{13}$.

\section{Une vision socio-pathologique des migrants}

"Que s'est-il passé à Cologne?", s'interroge justement Daoud, poursuivant : "On peine à le savoir avec exactitude en lisant les comptes rendus. " Dans les lignes qui suivent il précise : "mais on sait - au moins - ce qui s'est passé dans les têtes. Celles des agresseurs, peut-être; celles des Occidentaux, sûrement ». Avec élégance, Daoud mobilise le deuxième degré, celui - si puissant - des discours et des imaginaires. Mais n'ayant pas

Le débat est, au contraire,

tout de suite verrouillé

autour d'un argumentaire

qui récuse l'analyse détaillée

et circonstanciée des actes commis par des individus qui en portent la responsabilité

(éventuellement pénale)

en faveur d'une explication

centrée sur la « culture » des

agresseurs présumés. encore identifié les responsables des violences, comment pouvons-nous savoir ce qui s'est passé dans leurs têtes ? Comment en déduire l'antienne, qui semble rappeler le refrain de la droite plus ou moins fasciste qui fait rage en Europe : d'un côté des hommes non blancs (les " agresseurs »), de l'autre des hommes blancs (les « Occidentaux ") et, au milieu, des femmes blanches ('enjeu de l'affrontement). Un refrain que de nombreux journalistes et intellectuels non seulement répètent mais qu'ils contribuent à diffuser. Daoud en est bien évidemment conscient et sa lecture des violences de Cologne est toute centrée non pas sur le «fait " mais plutôt sur le « jeu d'images » qui permet de " délirer avec cohérence » (en français, le titre de l'article sur la Saint-Sylvestre est justement : "Cologne, lieu de fantasmes »). Sauf que Daoud semble se laisser emporter à son tour par le délire collectif. Et c'est dommage, parce que cela aurait été une occasion précieuse pour enfin se confronter avec ces fantasmes qui reviennent (perturbed spirit) déranger le sommeil européen.

Le débat est, au contraire, tout de suite verrouillé autour d'un argumentaire qui récuse l'analyse détaillée et circonstanciée des actes commis par des individus qui en portent la responsabilité (éventuellement pénale) en faveur d'une explication centrée sur la " culture » des agresseurs présumés. Nolens volens, Daoud donne une caution à cet argumentaire. "L'accueil [...] pêche en Occident par une surdose de naïveté ", écrit-il. "On voit, dans le réfugié, son statut, pas sa culture [...]. Sa culture est ce qui lui reste face au déracinement et au choc des nouvelles terres. Le rapport à la femme, fondamental pour la modernité de l'Occident, lui restera parfois incompréhensible pendant longtemps [...]. Il va donc en négocier les termes par peur, par compromis ou par volonté de garder "sa culture" ".

La seule chose que l'on sait des agresseurs de Cologne est qu'ils étaient sous l'emprise de l'alcool, et apparemment d'origine nord-africaine ou moyen-orientale. Cependant, après avoir fustigé le spectre du politiquement correct (la «naïveté » de l'accueil), l'auteur identifie sans hésitation les agresseurs dans la figure du "réfugié »: non pas «les réfugiés " au pluriel, compris comme des hommes et des femmes aux parcours, aux histoires et aux appartenances différentes (de l'Afghanistan à la Syrie), mais un coupable unique et clairement identifiable. Et ce « réfugié » - opaque, sans visage est emprisonné dans le piège de sa propre culture (la même pour tout le monde), celle du «monde d'Allah » (également appréhendé comme un monolithe sans nuances qui comprend un milliard et demi de personnes). "Le réfugié est-il donc un "sauvage" ?" Non, nous rassure l'écrivain ; il est seulement 
«malade ». Les violences de Cologne trouveraient en fait leur explication dans « le rapport pathologique que certains pays du monde arabe entretiennent avec la femme ", et qui désormais "fait irruption en Europe ", écrit Daoud dans un autre article publié dans The New York Times, où il précise : "Ce qui avait été le spectacle dépaysant de terres lointaines prend les allures d'une confrontation culturelle sur le sol même de l'Occident. Une différence autrefois désamorcée par la distance et un sentiment de supériorité est devenue une menace immédiate. Le grand public occidental découvre, dans la peur et l'agitation, que dans le monde musulman le sexe est malade et que cette maladie est en train de gagner ses propres terres ${ }^{14}$. "

En épousant sans équivoque la thèse du " clash culturel »-qui est aussi le fonds de commerce que se partagent l'extrême droite occidentale et Daesh -, Daoud semble finir par apporter sa caution à une guerre de fantasmes. Sans se rendre d'ailleurs compte - à l'instar de ses nombreux supporters, de droite comme de gauche - qu'il incarne lui-même la contradiction vivante de ce schéma binaire.

\section{Décrypter l'essentialisation des cultures}

Ce cohérent délire, passé d'abord inaperçu, a finalement poussé dix-neuf universitaires à signer une intervention critique publiée, toujours dans Le Monde, le 12 février : "Nuit de Cologne : "Kamel Daoud recycle les clichés orientalistes les plus éculés” ». Les dix-neuf - différents par le genre, l'âge, la position académique, l'origine (Algériens, Américains, Français, Italiens...) et le champ de recherche (histoire, anthropologie, philosophie, sociologie...), mais tous et toutes spécialistes du Maghreb et du Moyen Orient - protestent contre "une série de lieux communs navrants sur les réfugiés originaires de pays musulmans ». À leur avis, «loin d'ouvrir sur le débat apaisé et approfondi que requiert la gravité des faits, l'argumentation de Daoud ne fait qu'alimenter les fantasmes islamophobes d'une partie croissante du public européen, sous le prétexte de refuser tout angélisme ». Là aussi, il convient de revenir au plus près de la lettre du texte, tant par la suite la polémique a été âpre et abondamment alimentée par des fantasmes.

Les critiques principales que les dix-neuf adressent à l'écrivain sont au nombre de trois : essentialisme, psychologisation, discipline, des postures «typiques d'une approche culturaliste ».

Essentialisme, parce que Les critiques principales Daoud réduit un espace queles dix-neuf adressent extrêmement vaste et dif- àl'écrivain sont au nombre férencié "à une entité homo- de trois: essentialisme, gène, définie par son seul rap- psychologisation, discipline, port à la religion, "le monde des postures «typiques d'une d'Allah". Tous les hommes y approche culturaliste». sont prisonniers de Dieu et leurs actes sont déterminés par un rapport pathologique à la sexualité ». Comme si l'islam, de même que toute autre religion, ne s'articulait pas en un ample éventail de pratiques (nicodémiques, orthodoxes, fondamentalistes, opportunistes, libérales, mystiques...). «En miroir de cette vision asociologique qui crée de toutes pièces un espace inexistant, l'Occident apparaît comme le foyer d'une modernité heureuse et émancipatrice. La réalité des multiples formes d'inégalité et de violence faites aux femmes en Europe et en Amérique du Nord n'est bien sûr pas évoquée. Cet essentialisme radical produit une géographie fantasmée qui oppose un monde de la soumission et de l'aliénation au monde de la libération et de l'éducation. » Il s'agit d'un différend qui n'est pas sans rappeler la polémique suscitée par les thèses de Bernard Lewis, que certainement les dix-neuf ont à l'esprit au moment d'écrire leur critique ${ }^{15}$. Psychologisation, parce que les faits de Cologne se trouveraient expliqués par "l'état psychologique des masses musulmanes ", prisonnières "des discours islamistes ». "Psychologiser de la sorte les violences sexuelles est doublement problématique ", 
argumentent les dix-neuf. "D'une part, c'est effacer les conditions sociales, politiques et économiques qui favorisent ces actes (parlons de l'hébergement des réfugiés ou des conditions d'émigration qui encouragent la prédominance des jeunes hommes). D'autre part, cela contribue à produire l'image d'un flot de prédateurs sexuels potentiels, car tous atteints des mêmes maux psychologiques. Pegida n'en demandait pas tant. »

Enfin, discipline, parce que "culturellement inadaptés et psychologiquement déviants, les réfugiés doivent avant toute chose être rééduqués. Car Daoud ne se contente pas de diagnostiquer [...]. Selon lui, il faut "offrir l'asile au corps mais aussi convaincre l'âme de changer." C'est ainsi bien un projet disciplinaire, aux visées à la fois culturelles et psychologiques, qui se dessine ». Pour les dix-neuf, ce projet est "scandaleux ». "Au-delà du paternalisme colonial » qui l'imprègne, "il revient aussi à affirmer [...] que la culture déviante de cette masse de musulmans est un danger pour l'Europe. Il équivaut à conditionner l'accueil de personnes qui fuient la guerre et la dévastation ».

\section{Du fait divers à la polémique idéologique}

Cette controverse, pas foncièrement différente de beaucoup d'autres qui remplissent quasi quotidiennement les pages internes du Monde consacrées aux « débats », n'est cependant pas restée confinée dans le périmètre d'une escarmouche habituelle entre intellectuels. Bien au contraire, elle a alimenté une spirale d'hystérie collective qui a abouti à une campagne de véritable lynchage contre les dix-neuf, particulièrement après que Daoud ait annoncé sa volonté de quitter le journalisme pour se consacrer entièrement à la littérature. La décision - le journaliste l'admet lui-même - avait mûri plus tôt, avec les critiques de son ami et collègue américain Adam Shatz tâchant de le convaincre de prendre le chemin de l'Aventin ${ }^{16}$. Mais, dans l'espace public, s'est très vite imposée l'idée que la décision avait été prise suite à la tribune des dix-neuf : "L'intellectuel algérien: "Impossible d'écrire sur l'islam, je me retire" ", titrait le Corriere della Sera ${ }^{17}$.

L'écrivain, dans sa réponse à Shatz, n'hésite pas à dénoncer un véritable " procès stalinien » à son encontre tout en se moquant de ses détracteurs car ils sont " universitaires » et (donc) " planqués »: "Je trouve illégitime sinon scandaleux que certains me taxent d'être d'islamophobe à partir de la sécurité et du confort des capitales de l'Occident et de ses terrasses. "C'est ainsi que se répand l'idée que l'écrivain est victime d'une "fatwa médiatique18 ": l'article des dix-neuf universitaires équivaudrait donc à une menace de mort (comme celle prononcée par le salafiste Zeraoui à Alger) ou, dans le meilleur des cas, cela relèverait d'un geste intolérable de censure. Par exemple, Mohamed Mbougar Sarr écrit dans le Courrier International : "Kamel Daoud a su résister à une fatwa, mais c'est une tribune d'universitaires qui l'aura atteint ${ }^{19}$. Pas- $^{-}$ cal Bruckner, dans Le Monde, n’hésite pas à faire le parallèle avec la fatwa (tout sauf "médiatique » celle-ci) qui avait frappé Rushdie en 1989, comparant aussi « la fabrication planétaire d'un nouveau délit d'opinion analogue à ce qui se faisait, jadis, en Union soviétique contre les ennemis du peuple ${ }^{20}$ ». On retrouve les mêmes arguments chez Paul Berman et Michael Walzer, entre autres21. Pour Sophie Bélaïch (Huffington Post), qui jongle avec les armes de la critique et la critique des armes, "en visant Daoud, nous sommes tous en ligne de mire »: "Kamel Daoud, vous dérangez des prétendus bien-pensants malveillants qui se croient de gauche, mais qui encouragent clairement l'islamisme ${ }^{22} »$.

16. Kamel Daoud, "Lettre à un ami étranger ", in Quotidien d'Oran, 15 février 2016. Pour le portrait de Daoud : Adam Shatz, "Stranger Still. Kamel Daoud and Algeria, caught between Islamist fervor and cultural flowering ", in The New York Times, $1^{\text {er }}$ avril 2015. 17. Stefano Montefiori, "Intervista a Kamel Daoud. L'intelletuale algerino: Impossibile scrivere di Islam, mi ritiro ", in Corriere della Sera, 16 février 2016. 18. Alexandre Devecchio, "Kamel Daoud et la loi du silence », in Le Figaro, 22 février 2016.

19. Mohamed Mbougar Sarr, "Kamel Daoud victime de l'arrogance des universitaires », in Courrier international, 24 février 2016.

20. Pascal Bruckner, "Défendons les libres-penseurs contre les fatwas de l'intelligentsia ", in Le Monde, 2 mars 2016.

21. Paul Berman, Michael Walzer, "The Daoud affair. How Western intellectuals turn themselves into the enemies of an entire class of liberal writers from Muslim backgrounds ", in Tablet, 21 mars 2016. 22. Sophie Bélaïch, "Du silence coupable contre Kamel Daoud ", in Huffington Post, 24 février 2016. 
En très peu de jours, la tribune des dix-neuf déclenche une quantité de réactions, transversales dans l'échiquier politique, impressionnantes par leur nombre et surtout par leur violence. Les universitaires y sont taxés de tous les noms : «chiens de garde de la fatwa, déguisés en chercheurs » (Bruckner), «ignorants, malintentionnés voire complexés » (Bélaïch) qui ont « démoli " Daoud, "l'affublant d'un adjectif à la mode : islamophobe » (Michel Guerin ${ }^{23}$ ).

Même en Italie, hormis les rares exceptions habituelles (Internazionale, L'Indice ${ }^{24}$ ), on ne discute pas de Cologne et de la lecture qu'en a proposée l'écrivain algérien, mais de ses (et de nos) "fantasmes ». Pour Khaled Chaouki, député du Parti démocratique (gauche), les universitaires sont des " radicaux chics » qui propagent des «clichés angélistes », avant de conclure : «Il faut se débarrasser de l'hypocrisie du multiculturalisme ${ }^{25}$. "Dans les pages du Corriere della Sera, le quotidien le plus vendu du pays, la tâche de nous faire comprendre les éléments du débat revient à Susanna Tamaro : "Ce qui était autrefois une élimination physique de l'adversaire - le pilori, l'ordalie - devient maintenant un lynchage virulent dans les médias ${ }^{26}$. " Pour Mauro Zanon, sur MicroMega, Daoud - «nouveau martyr de la libre pensée " - serait «victime d'une "fatwa laïque" d'un groupe d'universitaires françai ${ }^{27}$ ».

L'acmé de cette campagne est probablement atteinte avec l'éditorial de Riss dans Charlie Hebdo. Dans la France post-attentats, le journal évolue sans entraves dans une xénophobie décomplexée, toujours bien évidemment sous la bannière de la bataille contre le "politiquement correct » (certains se rappelleront la vignette sur Cologne signée Riss : "Que serait devenu le petit Aylan s'il avait grandi ? Tripoteur de fesses en Allemagne $\left.{ }^{28} »\right)$. "Quand cette guerre contre l'islamisme sera terminée ", menace Riss dans les dernières lignes de l'éditorial du 24 février, «il faudra faire les comptes de toutes les lâchetés, les complaisances, les trahisons des intellos et des journalistes qui auront tout fait pour intimider et faire taire les voix contestataires ${ }^{29}$ ". Il n'est pas du tout évident de savoir ce que Riss entend par "faire les comptes ", mais cela ne semble promettre rien de rassurant.

Désormais, il apparaît clairement que le nœud de la discorde n'est pas ce qui s'est passé la nuit de la Saint-Sylvestre à Cologne, ni la lecture qu'en a donnée Daoud, qui constate quelque temps après : "Dans l'affaire "Cologne", j'ai fini par comprendre que je n'étais que le déclencheur de quelque chose qui couvait et qui attendait ${ }^{30}$. » Pour confirmer le fait que la bataille se passe ailleurs, intervient le Premier ministre Manuel Valls qui exprime sur Facebook son soutien inconditionné à l'écrivain : "Les attaques, la hargne inouie dont Kamel Daoud fait l'objet depuis quelques jours ne peuvent que nous interpeller, nous indigner. Et pour tout dire : nous consterner. » «Certains universitaires », continue le Premier ministre sans trop se soucier de la réalité, « au lieu d'éclairer, de nuancer, de critiquer [...], condamnent de manière péremptoire, refusent le débat et ferment la porte à toute discussion. Le résultat est connu : un romancier de talent - et sur qui pèse déjà une "fatwa" dans son pays - décide, face à la violence et à la puissance de la vindicte, de renoncer à son métier de journaliste. C'est tout simplement inconcevable ${ }^{31}$. »

\section{Une juste distance devant l'événement}

En Algérie, la presse s'est saisie de l'affaire d'une manière significativement plus articulée et lucide. La presse arabophone, de son côté, est restée plutôt distante. El Khabar a d'abord laissé Ismail 
Mehnana, professeur de philosophie à l'université de Constantine, affirmer que Daoud était tombé dans une "généralisation fausse, réductrice et hâtive "; quelques jours plus tard, le quotidien se débarrassait de l'" affaire " en insinuant que "la critique des "dix-neuf" a largement contribué à [la] reconnaissance [de Daoud] en France, et c'est peut-être ce que voulait l'écrivain... ${ }^{32}$ ». De son côté, la presse francophone surprend, surtout après avoir parcouru le monologue collectif distillé en une centaine d'articles dans les quotidiens européens et nord-américains.

L'« affaire de Cologne»,

à y regarder de plus près, montre bien ces logiques discursives - politiques et idéologiques - qui président

à la fabrication de notre présent postcolonial.
Pour Aziz Benyahia (Algeria Focus), si les critiques des dix-neuf peuvent paraître excessives, elles " reposent cependant sur des arguments qui sont recevables. Et ces accusations ne sont pas injustifiées, compte tenu du climat d'hostilité quasi générale dans lequel se débat la communauté musulmane. Quand le monde entier épie les moindres faits et gestes des musulmans et de ces hordes d'assassins qui se réclament de l'islam, on est légitimement en droit d'attendre un peu plus de circonspection et à tout le moins, plus de nuances dans l'expression de la part de ceux qui sont censés séparer le bon grain de l'ivraie, par la pertinence de leurs analyses et au nom de leur liberté dans l'expression ${ }^{33}$ ". Enfin, comme l'a reconnu un des "dix-neuf ", Thomas Serres, la forme du texte collectif était très mal choisie: « les textes collectifs fonctionn[ent] bien mieux contre des institutions ou des États » que contre un individu isolé ${ }^{34}$. Et pourtant, l'analyse de la nuit de Cologne proposée par Daoud - bien que lue en Algérie avec émotion et attention - ne manque pas de susciter « la déception, puis la colère et l'indignation, le sentiment d'avoir été trahis par l'un des nôtres. Comment se tromper à ce point?", demande Ahmed Bacha ${ }^{35}$.

Hacen Ouali, dans El Watan, dénonce le «lynchage religieux et politico-médiatique sans pareil de Daoud", mais il s'attire une réponse longue et détaillée de son collègue Ahmed Cheniki dans les pages du Matin $^{36}$. Selon lui, la fable des "dix-neuf », tout compte fait d'obscurs universitaires qui avec une tribune d'à peine 900 mots font faire taire un écrivain à la renommée mondiale, ne tient pas debout. C'est une offense à l'intelligence : "Ce qui est reproché à Kamel Daoud, et [les dix-neuf] ont le droit de le dire, c'est le discours essentialiste développé dans ses deux chroniques des quotidiens, Le Monde et The New York Times opposant deux constructions idéologiques, "'Occident" et le "Monde arabo-musulman". J'aime beaucoup ce que disent Edward Saïd, Malek Alloula et Mohammed Dib qui s'insurgent contre ceux qui fabriquent leur "monde musulman", "leur monde noir", à l'aide de stéréotypes, comme si cétait un bloc monolithique, évacuant les nombreuses différences, les dizaines de langues et les réalités sociologiques et anthropologiques ${ }^{37}$. ». Dans ce schéma binaire, en fait, il n'y a pas de place pour les réfugiés qui, le 16 janvier 2016, ont manifesté à Cologne contre la violence, le sexisme et le racisme ni pour ceux qui, selon les témoignages, ont aidé certaines femmes à échapper aux griffes des agresseurs : c'est le cas, entre autres, de l'étudiante américaine Caitlin Duncan, qui a été protégée par un groupe de Syriens ${ }^{38}$. D'ailleurs, comme l'ont fait remarquer de nombreuses féministes, descendues dans la rue à Cologne le jour suivant celui des violences (et totalement ignorées par les médias), «il n'y a pas de monopole islamique sur la violence et l'infériorisation des femmes. Et il n'y a même pas de monopole occi-

32. "Daoud a affronté une réalité difficile avec des conceptions orientalistes de l'islam ", in El Khabar, 26 février 2016 ; "Condamnation de Hamadashe à six mois de prison dont trois ferme ", in El Khabar, 8 mars 2016. 33. Aziz Benyahia, "Notre solidarité sans faille avec Kamel Daoud ", in Algeria Focus, 19 février 2016. 34. Thomas Serres, "Autopsie d'une défaite et notes de combat pour la prochaine fois ", in Article 11, 2 mars 2016. Une autre des dix-neuf a répondu aux critiques : Jocelyne Dakhlia, "Faire croire à un choc des cultures, voilà la vraie défaite du débat ", in Le Monde, 2 mars 2016. 35. Ahmed Bacha, "Et puis il y eut Cologne,... ", in Le Matin, 21 février 2016.36. Hacen Ouali, "Les nouveaux procureurs de la pensée... ? ", in El Watan, 22 février 2016 ; Ahmed Cheniki, «À propos d'un article d'El Watan sur la polémique autour de Kamel Daoud », in Le Matin, 24 février 2016. 37. Ibid. 38. Voir Alison Smale, "In new year's melee in Cologne, a migrant was one women's savior ", in The New York Times, 15 janvier 2016 
dental et démocratique de la liberté féminine ${ }^{39}$ ». Les viols se produisent tous les jours, partout. Selon une étude de l'Agence pour les droits fondamentaux de l'Union européenne (2014), 62 millions de femmes en Europe (33 \% de la population féminine) déclarent avoir été l'objet d'une forme de violence à connotation sexuelle ${ }^{40}$. En 2016, en Italie (mais c'est à peu près la même chose en France), 116 femmes ont été tuées par leur mari, fiancé, compagnon ou par des membres de leur famille: cela fait une tous les trois jours, mais on n'a pas expliqué cette guerre silencieuse par la culture italo-catholique. La " culture », c'est pour les Autres.

\section{Conclusion}

Il semblerait finalement que les "fantasmes » de Cologne ne concernent pas tant un "sexisme oriental » (qui bien sûr existe) mais plutôt la réactualisation, sur le corps des femmes allemandes agressées, du dispositif rhétorique qui était au cœur du projet colonial : "des hommes blancs qui sauvent des femmes de couleur des hommes de couleur ", selon la célèbre définition de Gayatri Spivak ${ }^{41}$.

"Kamel Daoud ", écrit Ahmed Cheniki, "oppose deux logiques, deux totalités bâties sur l'exclusion d'origine essentialiste : celle d'un monde occidental parfait, heureux, moderne et celle d'une entité arabomusulmane, masse informe, archaïque, vivant un rapport maladif à la femme et à la sexualité. L'Arabomusulman, une catégorie informe, unique, sans possibilité d'autonomie est un "zombie", un obsédé sexuel qui devrait être soigné, subir une radicale désintoxication purificatrice avant d'être admis dans le monde de la pureté occidentale. Ce manichéisme est dangereux parce qu'il évacue toute dimension humaniste et toute identité métisse, hybride, faite de traces culturelles multiples. Qu'est-ce qu'un "Arabo-musulman"? Qu'est-ce qu'un "Occidental" ? Ce ne sont que des simples constructions idéologiques mises en ouvre pour légitimer un illusoire choc des civilisations ${ }^{42}$. ” Frantz Fanon, conclut Cheniki, disait à propos de la torture : "Le peuple européen qui torture est un peuple déchu, traître à son histoire. Le peuple sous développé qui torture assure sa nature, fait son travail de peuple sous-développé. »

L'« affaire de Cologne ", à y regarder de plus près, montre bien ces logiques discursives - politiques et idéologiques - qui président à la fabrication de notre présent postcolonial. C'est-à-dire à l'invention d'un monde figé, divisé en deux entités molaires racialisées, blocs culturels inconciliables et sans nuances, où les hommes et les femmes en chair et en os disparaissent derrière l'écran d'un choc des civilisations fantasmé. Une vision des choses où vont paradoxalement se retrouver, bras dessus bras dessous, les croisés défenseurs de l'« Occident » et les combattants d'un «djihad » globalisé. S'apercevoir du piège de l'«identité " ne revient pas à nier la complexité des défis que nous avons en face de nous, ni à céder à la dystopie d'un monde sans conflits (il serait d'ailleurs intéressant, par exemple, d'étudier l'impact du porno sur l'image que les jeunes migrants se font de la « sexualité occidentale », la pornographie sur Internet étant sans doute notre première marchandise culturelle d'exportation). Cela revient plutôt à comprendre que toute bataille pour la liberté est aussi une bataille pour la connaissance, et la question n'est donc plus de savoir ce qu'est l'« identité », mais plutôt comment celle-ci fonctionne. 